\title{
Electric field measurements on Cluster: comparing the double-probe and electron drift techniques
}

\author{
A. I. Eriksson ${ }^{1}$, M. André ${ }^{1}$, B. Klecker ${ }^{2}$, H. Laakso ${ }^{3}$, P.-A. Lindqvist ${ }^{4}$, F. Mozer ${ }^{5}$, G. Paschmann ${ }^{2,6}$, A. Pedersen ${ }^{7}$, \\ J. Quinn ${ }^{8}$, R. Torbert ${ }^{8}$, K. Torkar ${ }^{9}$, and H. Vaith ${ }^{2,8}$ \\ ${ }^{1}$ Swedish Institute of Space Physics, Uppsala, Sweden \\ ${ }^{2}$ Max-Planck-Institut für Extraterrestrische Physik, Garching, Germany \\ ${ }^{3}$ Solar System Division, ESA/ESTEC, Noordwijk, Netherlands \\ ${ }^{4}$ Alfvén laboratory, Royal Institute of Technology, Stockholm, Sweden \\ ${ }^{5}$ Space Sciences Laboratory, University of California, Berkeley, CA, USA \\ ${ }^{6}$ International Space Science Institute, Bern, Switzerland \\ ${ }^{7}$ Department of Physics, Oslo University, Norway \\ ${ }^{8}$ Space Science Center, University of New Hampshire, Durham, NH, USA \\ ${ }^{9}$ Space Research Institute, Austrian Academy of Sciences, Graz, Austria
}

Received: 16 June 2004 - Revised: 29 November 2005 - Accepted: 14 December 2005 - Published: 7 March 2006

\begin{abstract}
The four Cluster satellites each carry two instruments designed for measuring the electric field: a doubleprobe instrument (EFW) and an electron drift instrument (EDI). We compare data from the two instruments in a representative sample of plasma regions. The complementary merits and weaknesses of the two techniques are illustrated. EDI operations are confined to regions of magnetic fields above $30 \mathrm{nT}$ and where wave activity and $\mathrm{keV}$ electron fluxes are not too high, while EFW can provide data everywhere, and can go far higher in sampling frequency than EDI. On the other hand, the EDI technique is immune to variations in the low energy plasma, while EFW sometimes detects significant nongeophysical electric fields, particularly in regions with drifting plasma, with ion energy (in $\mathrm{eV}$ ) below the spacecraft potential (in volts). We show that the polar cap is a particularly intricate region for the double-probe technique, where large nongeophysical fields regularly contaminate EFW measurments of the DC electric field. We present a model explaining this in terms of enhanced cold plasma wake effects appearing when the ion flow energy is higher than the thermal energy but below the spacecraft potential multiplied by the ion charge. We suggest that these conditions, which are typical of the polar wind and occur sporadically in other regions containing a significant low energy ion population, cause a large cold plasma wake behind the spacecraft, resulting in spurious electric fields in EFW data. This interpretation is supported by an analysis of the direction of the spurious electric field, and by showing that use of active potential control alleviates the situation.
\end{abstract}

Correspondence to: A. I. Eriksson

(anders.eriksson@irfu.se)
Keywords. Magnetospheric physics (Electric fields; Instruments and techniques) - Space plasma physics (Spacecraft sheaths, wakes, charging)

\section{Introduction}

The electric field is a key parameter for determining and modelling various space plasma physics processes, for example, reconnection and particle acceleration. Modern spacecraft for in-situ studies of space plasma physics, therefore, usually carry instruments for observing the electric field, from zero frequency up to frequencies well above the highest characteristic frequencies in the plasma. For measurement of low frequency and quasi-static fields in low density plasmas, the two main measurement techniques employ double probes and electron drift instruments. Descriptions of these techniques are provided by Pedersen et al. (1998) and Maynard (1998) for double probes, and by Paschmann et al. (1998) for electron drift instruments. In brief, the operational principle of a double probe instrument is to measure the voltage difference between two usually spherical probes, which, for magnetospheric conditions, must be forced to stay close to the potential of the unperturbed plasma at their respective positions by use of a suitably chosen bias current (Fig. 1). The electron drift technique is based on the fact that to zeroth order, gyrating charged particles drift at a velocity $\boldsymbol{E} \times \boldsymbol{B} / B^{2}$. This drift velocity can be determined using two properly directed electron beams which must be detected upon their return to the spacecraft (Fig. 2). The equivalent electric field can then be calculated from the drift and from magnetic field data. 


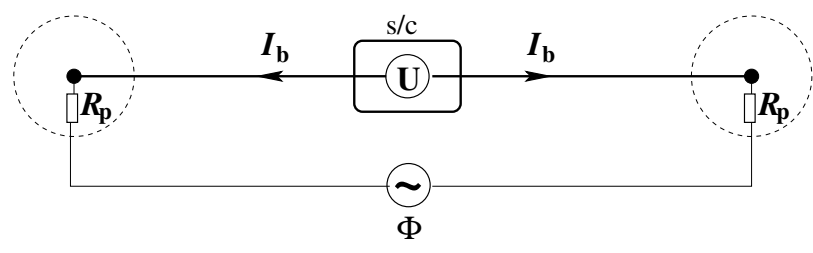

Fig. 1. The operational principle of a double probe instrument. Two boom-mounted probes (solid circles) are fed with identical bias currents $I_{\mathrm{b}}$. If the resistances $R_{\mathrm{p}}$ over the probe sheaths (dashed circles) are equal, the voltage $U$ measured on board the spacecraft will be equal to the potential difference $\Phi$ in the plasma between the probe locations. Unwanted electric field signals can arise either from a difference in $R_{\mathrm{p}}$ between the probes, or from an asymmetric potential structure around the spacecraft and booms adding to the unperturbed $\Phi$ in the plasma. Currents close through the spacecraft sheath (not shown). For a more complete description, see Pedersen et al. (1998).

Each technique has its own merits and weaknesses. Double probe instruments have relative advantages in terms of conceptual simplicity, regular and essentially unlimited sampling frequency, the possibility to measure rapidly varying fields at arbitrarily high amplitudes, and an operational principle independent of the magnetic field. On the other hand, as the measurement principle depends on the electrostatic coupling of the probe to the plasma surrounding it, the technique is sensitive to perturbations from the spacecraft or the wire booms supporting the probes. Though there are many ways to reduce such perturbations, including design symmetry, biasing of probes and bootstrapping of adjacent boom elements, their possible influence always constitutes an uncertainty which only comparison to other measurements can eliminate. In contrast, electron drift instruments are quite insensitive to the details of the spacecraft environment, as the keV energy typical for electrons emitted by EDI is much higher than any potentials arising on a well-designed scientifc spacecraft (normally less than $50 \mathrm{~V}$ ). In the weak magnetic fields typical for Cluster, the emitted electrons also spend most of their time in an orbit far away from the spacecraft, further diminishing any influence of the spacecraft-plasma interaction. In addition, the electron drift technique does not depend on spacecraft orientation, while double probe instruments at best can have shorter booms along the spin axis and are often confined to measurements in the spin plane. A strength of the EDI technique is that the measurement relies upon simple geometry; thus, when beam tracking is successful the absolute measurement is relatively reliable and does not require calibration or offset correction. However, as the electron drift method relies on observing electrons returned to the spacecraft by the ambient magnetic and electric fields, the magnetic field has to be sufficiently strong for the emitted beam not to disperse too much for detection. Rapid variations in the magnetic or electric field will also complicate the beam tracking, so the method works best in regions where the field variations are less rapid than the tracking bandwidth $(\sim 100 \mathrm{~Hz})$, and the angular step-

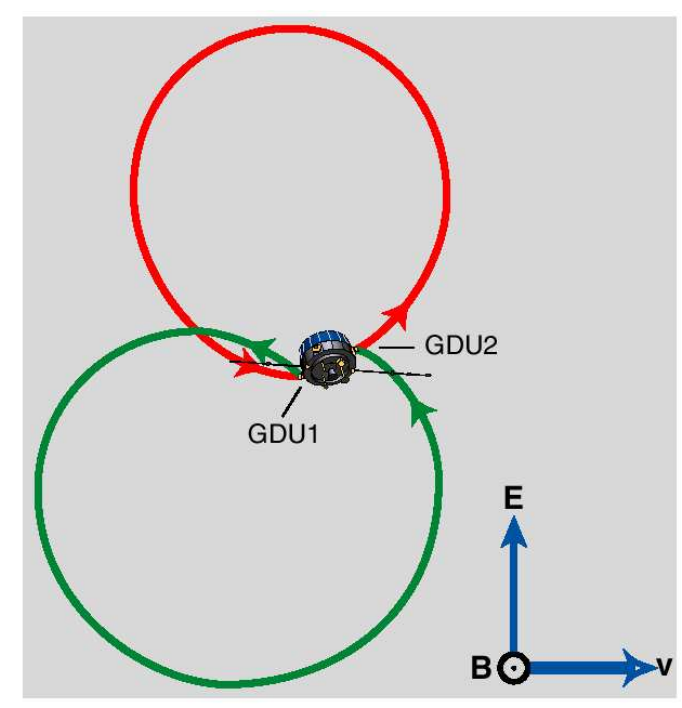

Fig. 2. The operational principle of the EDI electron drift instrument on Cluster, using two gun-detector units (GDU1 and GDU2) emitting two beams of keV electrons and detecting their drift upon return. For any given magnetic field $\boldsymbol{B}$ and drift velocity $\boldsymbol{v}$, here assumed to be solely due to an electric field $\boldsymbol{E}$, only one orbit exists that connects each gun with the opposite detector, enabling a unique determination of $\boldsymbol{v}$ and hence of $\boldsymbol{E}$. The drawing is not to scale: in a 100-nT magnetic field, the orbits of the EDI electrons reach $2 \mathrm{~km}$ from the spacecraft. For details, see Paschmann et al. (2001).

ping rate of the beam. Furthermore, sufficiently strong ambient electron fluxes near the beam energy (typically $1 \mathrm{keV}$ ) can swamp the beam signal and prevent detection. Table 1 summarizes the performance of the two techniques.

As the strengths and limitations of the two techniques are so different, they complement each other. Each of the four Cluster spacecraft (Escoubet et al., 2001), therefore, carries one instrument of each type: the double-probe instrument EFW (Electric Fields and Waves, Gustafsson et al., 1997, 2001) and the Electron Drift Instrument (EDI, Paschmann et al., 1997, 2001). Since the start of nominal operations in February 2001, EFW has operated on all four spacecraft essentially all the time. Though EDI operations are restricted to regions with a sufficiently intense magnetic field, and were in use on Cluster spacecraft four (Tango) only briefly, there is a large amount of simultaneous data from the two instruments available for comparison. In addition, the EDI implementation flying on Cluster is of a design very much improved over previous missions, so there are unprecedented possibilities to compare the performances. Finally, data obtained by both techniques are made widely available to the scientific community through the Cluster Science Data System (Daly, 2002), so there is also an unprecedented need to compare the data in order to provide a background for users of Cluster electric field data. This is the scope of the present study. We cannot exhaust all pitfalls and limitations of either technique in one single paper, but we aim at illustrating the general features, particularly pointing out discrepancies arising especially over the polar caps. 
Table 1. Summary of merits and drawbacks of the double-probe and electron drift techniques for magnetospheric electric field measurments. The implementations on Cluster, EFW and EDI, provide 2-D measurements. Extending the EDI technique to three-dimensional measurements will require a significant advance over the current state of the art.

\begin{tabular}{|c|c|c|}
\hline & Double-probes (EFW) & Electron drift (EDI) \\
\hline Ambient $B$ & no limitations & $\gtrsim 30 \mathrm{nT}$ on Cluster \\
\hline Frequency range & DC to $\mathrm{MHz}$ & $\lesssim 10 \mathrm{~Hz}$ (depending on beam returns) \\
\hline Dimensionality & 2-D (spin plane) or 3-D (if axial booms) & 2-D $(\perp \boldsymbol{B})$ or 3-D (in principle) \\
\hline Sensitivity to thermal/cold plasma & Yes & No \\
\hline Sensitivity to s/c-plasma interactions & Yes & No \\
\hline Sensitivity to ambient keV electrons & Low & May swamp signal \\
\hline Sensitivity to B-field variations & No & Yes \\
\hline Additional data products & S/c potential, plasma density, waves & B-field magnitude \\
\hline Alternative data products & $\begin{array}{l}\text { Density and temperature } \\
\text { from use as Langmuir probe }\end{array}$ & $\begin{array}{l}\mathrm{keV} \text { electron measurements } \\
\text { at high time resolution }\end{array}$ \\
\hline
\end{tabular}

It should be noted that in this paper we concentrate on the two Cluster instruments specifically designed for obtaining electric field measurements, i.e. EDI and EFW. One may also construct an electric field estimate from the velocity moment $\boldsymbol{v}_{\mathrm{i}}$ from the Cluster ion spectrometers (CIS, Rème et al., 2001 ) and the magnetic field $\boldsymbol{B}$ from the FGM fluxgate magnetometers (Balogh et al., 2001), assuming $\boldsymbol{E}+\boldsymbol{v}_{\mathrm{i}} \times \boldsymbol{B}=0$. We will use this to obtain a "third opinion" on the electric field in cases where EFW and EDI disagree, and we will also include some CIS and FGM data for establishing the geophysical context of the data we show, but a complete CIS-EFW comparison, also in regions where there are no EDI data, is outside the scope of the present study, as is any details of measurement errors in the CIS data.

While the Cluster data set for comparison of the two techniques surpasses what is available from previous missions, we should note that some comparative studies have been made before. Bauer et al. (1983) and Pedersen et al. (1984) compared data from the two instrument types on the GEOS satellites, finding some effects that we will also see in Cluster data. Kletzing et al. (1994) showed data from the F1 (double probes) and F6 (electron drift) instruments on the Freja satellite in the topside ionosphere. Finally, the Geotail satellite carries instruments of both kinds, allowing Tsuruda et al. (1994) to compare their initial results.

\section{EDI-EFW comparison in various plasma regions}

\subsection{Example 1: Solar wind-magnetosheath-plasma mantle}

\subsubsection{Geophysical setting}

Our first example spans $12 \mathrm{~h}$, from 12:00 to 24:00 UT, on 13 February 2001. The orbit of Cluster during this time interval is illustrated in Fig. 3. As can be seen from the model boundaries and field lines in this figure, Cluster should move from the solar wind through the magnetosheath and into the magnetosphere during this time interval. The entry into the magnetosphere occurs duskward of the southern cusp, so that Cluster at the end of the interval is on field lines reaching the duskside plasma mantle or low-latitude boundary layer.

Figure 4 shows $12 \mathrm{~h}$ of data from Cluster SC3. The top three panels $(\mathrm{a}-\mathrm{c})$ show the electric field measurements that are our real topic here and to which we will return after describing the geophysical setting. The lower three panels (df) are auxilliary data for illustration of the plasma environments. As expected from Fig. 3, the spacecraft was in the solar wind at the start of the interval (13 February 2001, 12:00 UT), with weak magnetic field (FGM data, bottom panel f) and a density around $10 \mathrm{~cm}^{-3}$ (CIS HIA density moment, panel e). The first bow shock crossing can be seen around 14:40 UT, with an increase in density and magnetic field. The increasing density causes the electrostatic potential of the spacecraft with respect to the surrounding plasma, $V_{\mathrm{sc}}$, to decrease, as more plasma electrons become available for compensating the emission of photoelectrons. This is seen as a small increase in the EFW probe-to-plasma potential, $V_{\mathrm{ps}}$, which essentially is the negative of $V_{\mathrm{sc}}$ and thus will covary with the density. One may therefore use $V_{\mathrm{ps}}$ as a proxy for the plasma density. How to convert from $V_{\mathrm{ps}}$ to plasma density has been reported for Cluster by Pedersen et al. (2001).

The magnetopause is crossed around 20:10 UT, after which the magnetic field (panel (f) of Fig. 4) increases as the spacecraft comes closer to the Earth. In the plasma mantle, the density as reported from CIS HIA (panel e) and EFW $V_{\mathrm{ps}}$ decreases monotonically to reach the limit of the HIA instrument sensitivity just after 22:00 UT. After this time, the $V_{\mathrm{ps}}$ data indicate a density increase not noted by the HIA ion spectrometer, which is the expected behaviour if the ion energy is below the spacecraft potential, so that the ions cannot reach the particle instrument. ${ }^{1}$ The $V_{\mathrm{ps}}$ data suggest a density increase towards $1 \mathrm{~cm}^{-3}$ at the end of the interval at 24:00 UT. As the impact of this population is seen in the spacecraft potential but not in the ion detector, the ion energy

\footnotetext{
${ }^{1}$ For convenience, we implicitly assume normalization to the elementary charge $e$ whenever comparing energies and potentials.
} 

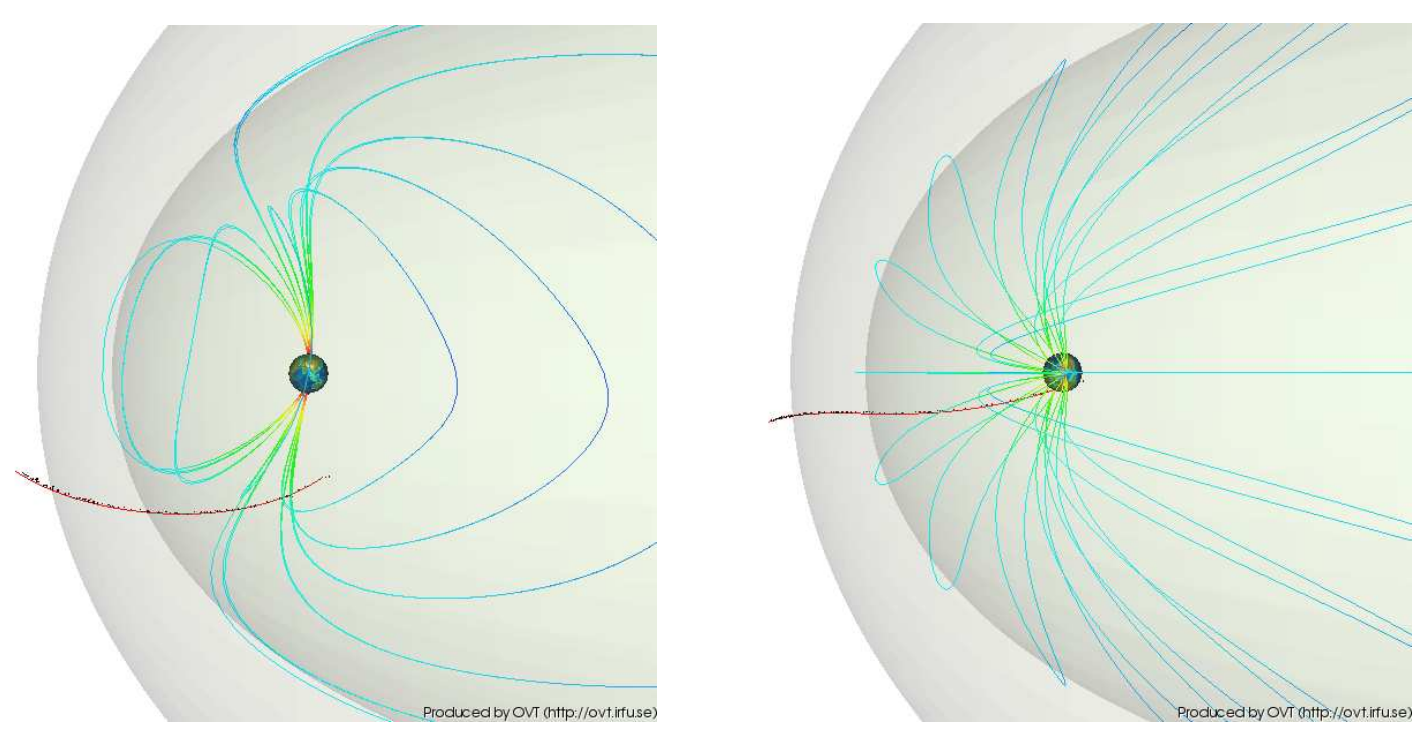

Fig. 3. Cluster orbit (red) for 13 February 2001, corresponding to the data in Fig. 4, viewed from GSE Y (left) and Z (right) directions. Model magnetosheath (light shading) and magnetosphere (dark shading) are shown, as are some magnetic field lines colour coded for magnetic field intensity. Cluster moves inbound, from the solar wind to the magnetosphere. Plot prepared using the Orbit Visualization Tool, http://ovt.irfu.se.
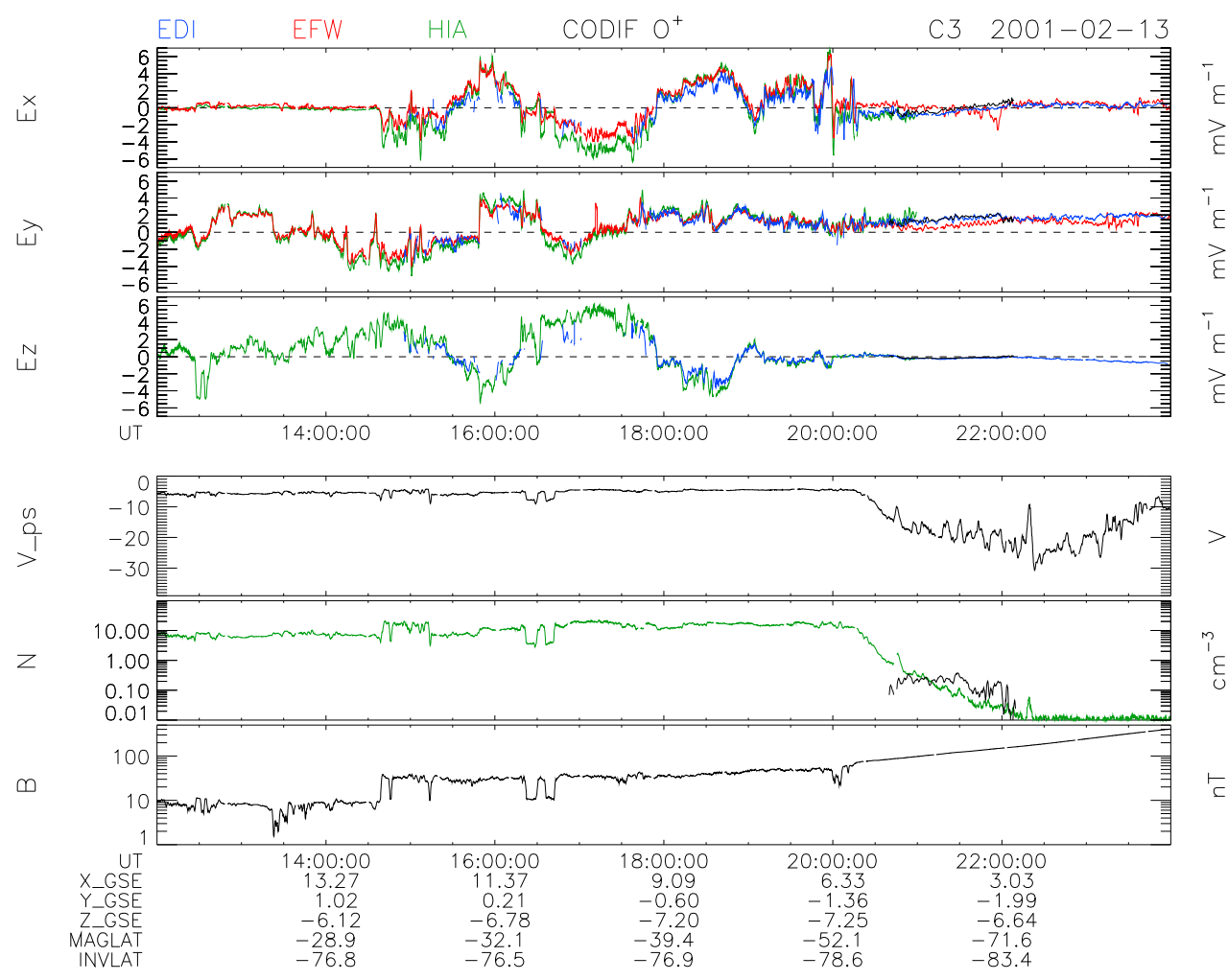

Fig. 4. Comparison of EFW and EDI data from the solar wind through the magnetosheath into the magnetosphere. Panels from top to bottom: (a) $E_{x}$ in DSI coordinates (almost GSE, see text) in an inertial frame. Red is EFW data based on spin fits from probe pair 12, blue is EDI, black is the $x$ component of $-\boldsymbol{v} \times \boldsymbol{B}$ from CIS HIA velocity moment and FGM magnetic field, green is the same for CIS CODIF $\mathrm{O}^{+}$data. (b, c) $E_{y}$ and $E_{z}$ in same format. (d) EFW probe-to-spacecraft potential $V_{\mathrm{ps}} \approx-V_{\mathrm{sc}}$. (e) Density moments from CIS: HIA (green, assuming protons only) and $\mathrm{CODIF} \mathrm{O}^{+}$(black). (f) FGM magnetic field magnitude. 
must stay at least below $25 \mathrm{eV}$ after 22:00 UT, and below $10 \mathrm{eV}$ close to midnight. One may also note that this cold plasma shows quite a lot of structure.

The top three panels in Fig. 4 show the electric field measurements by EFW (red) and EDI (blue), and the electric field inferred from the FGM magnetic field measurements and the velocity moments $\boldsymbol{v}_{\text {i }}$ from the CIS HIA (green) or CODIF (oxygen ions, black) detectors, assuming $\boldsymbol{E}+\boldsymbol{v}_{\mathrm{i}} \times \boldsymbol{B}=0$. The EFW data shown are deduced by fitting a sinusoidal function to the voltage between probes 1 and 2 . We have not corrected the EFW data for any well-known effects in double-probe instruments, like sunward offsets or partial shielding (Pedersen et al., 1998; Maynard, 1998). The impact of such corrections will instead be discussed as data are presented.

To transform CIS velocity moments, we assume that $\boldsymbol{E}+\boldsymbol{v}_{\mathrm{i}} \times \boldsymbol{B}=0$. We have chosen to include only EFW measurements from the plane in which these are made, i.e. the spin plane, though, in principle, the third component could be derived using magnetometer data and $\boldsymbol{E} \times \boldsymbol{B}=0$ as an assumption. All data are therefore given in a reference frame known as despun inverted, or DSI, coordinates, which is a close approximation to GSE but with the $\mathrm{Z}$ axis along the spacecraft spin axis. If the spacecraft spin was exactly aligned with the GSE $Z$ axis, the DSI and GSE systems would be identical: for Cluster they differ only by a few degrees.

It should be noted that all the methods used to determine the electric field signals in this plot, in fact, are twodimensional, either by being utilized in the spin plane (EFW) or in the plane perpendicular to $\boldsymbol{B}$ (EDI), or by assuming $\boldsymbol{E}+\boldsymbol{v}_{\mathrm{i}} \times \boldsymbol{B}=0$ (CIS). Three-dimensional double-probe electric field measurements have been implemented on other spacecraft, for example, on the Polar EFI instrument (Harvey et al., 1995), using shorter axial booms, and could, in principle, also be implemented by an EDI technique.

\subsubsection{Solar wind}

In the weak magnetic field in the solar wind, i.e. before 14:40 UT in Fig. 4, EDI cannot provide data, but it is clear that EFW and CIS agree to well within a $\mathrm{mV} / \mathrm{m}$. While comparison of EFW and CIS data is not a prime topic in this paper, we may note in passing that this agreement is typical for spin resolution data in the solar wind, though velocity wakes may at times contaminate the sunward component in higher resolution EFW data, as may be expected in the supersonically flowing solar wind. A detailed scrutiny will show some tendency, seen most clearly in the $E_{Y}$ data in panel (b), for the EFW electric field, to show slightly lower magnitude than expected from CIS velocity. This can be attributed to the effective antenna length being slightly shorter than the physical probe separation, due to the effect of the conductive wire booms on the real electric field. In effect, the booms partially short-circuit or shield away the ambient electric field. This effect is well-known (e.g. Mozer, 1973; Pedersen et al., 1998) and results in underestimates of the E-field magnitude of some $20 \%$ for Cluster EFW in tenuous plasmas. In panel (a), showing the sunward component $E_{X}$, the effect is partially masked by a close-to-constant sunward offset field of $0.5 \mathrm{mV} / \mathrm{m}$. The sunward offset is due to the inevitable photoemission asymmetry between probes on booms pointing toward and away from the Sun (Pedersen et al., 1998). In the following discussion of other events, we will not further comment on the sunward offset or the partial shielding, but the reader should be aware that these effects always influence double-probe electric field data to some extent.

\subsubsection{Magnetosheath}

After having entered the magnetosheath, the first time close to 14:40 UT (Fig. 4), EDI data starts appearing intermittently when the magnetic field strength is sufficiently high, the limit typically being around $30 \mathrm{nT}$. When present, EDI data agrees well with EFW and CIS in this region, despite EDI obviously operating close to and sometimes below its low- $B$-field limit. An exception is the large $E_{X}$ just before 20:00 UT, occurring in a region of enhanced magnetic activity (not shown) which complicates the interpretation of EDI data. CIS shows deviations from EDI and EFW, particularly in $E_{X}$, around 15:00 and 17:00 UT, where the differing values derived from CIS HIA are due to instrumental reasons outside the scope of this paper. For EFW, the magnetosheath usually is a relatively benign region, as the Debye length normally is well below the boom length and the plasma flow is subsonic, thus not creating appreciable wakes.

\subsubsection{Plasma mantle}

Following the spacecraft into the magnetosphere from 20:10 UT onwards (Fig. 4), we expect the conditions to become more suited to the EDI measurement technique as the background magnetic field becomes stronger and less variable than in the magnetosheath. This is confirmed by the good agreement we find between EDI and CIS ion data. For the CIS data, the velocity moment after about 21:00 UT must be calculated from the mass-separated data from the CODIF sensor because of the increased relative abundance of oxygen. One should note that even though the behaviour of the spacecraft potential shows that the ion detectors only capture a fraction of the ion population, the ion velocity moment should still be reasonably reliably determined as long as there is a sufficient count rate, particularly when using mass-separated data. We thus conclude that EDI works well in this region of the magnetosphere.

While EDI and CIS agree well in the mantle, i.e. after 20:10 UT, we start seeing some hints of EFW slightly deviating. The discrepancy is small in this example, around a $\mathrm{mV} / \mathrm{m}$, except for the spike in $E_{X}$ at 22:00 UT. We believe the cause of the deviation is to be found in the effects of the cold plasma component discussed above in Sect. 2.1.1. Similar discrepancies will be encountered in some other environments presented below, but are most pronounced in the polar cap region. We will discuss them in detail in Sect. 3. 

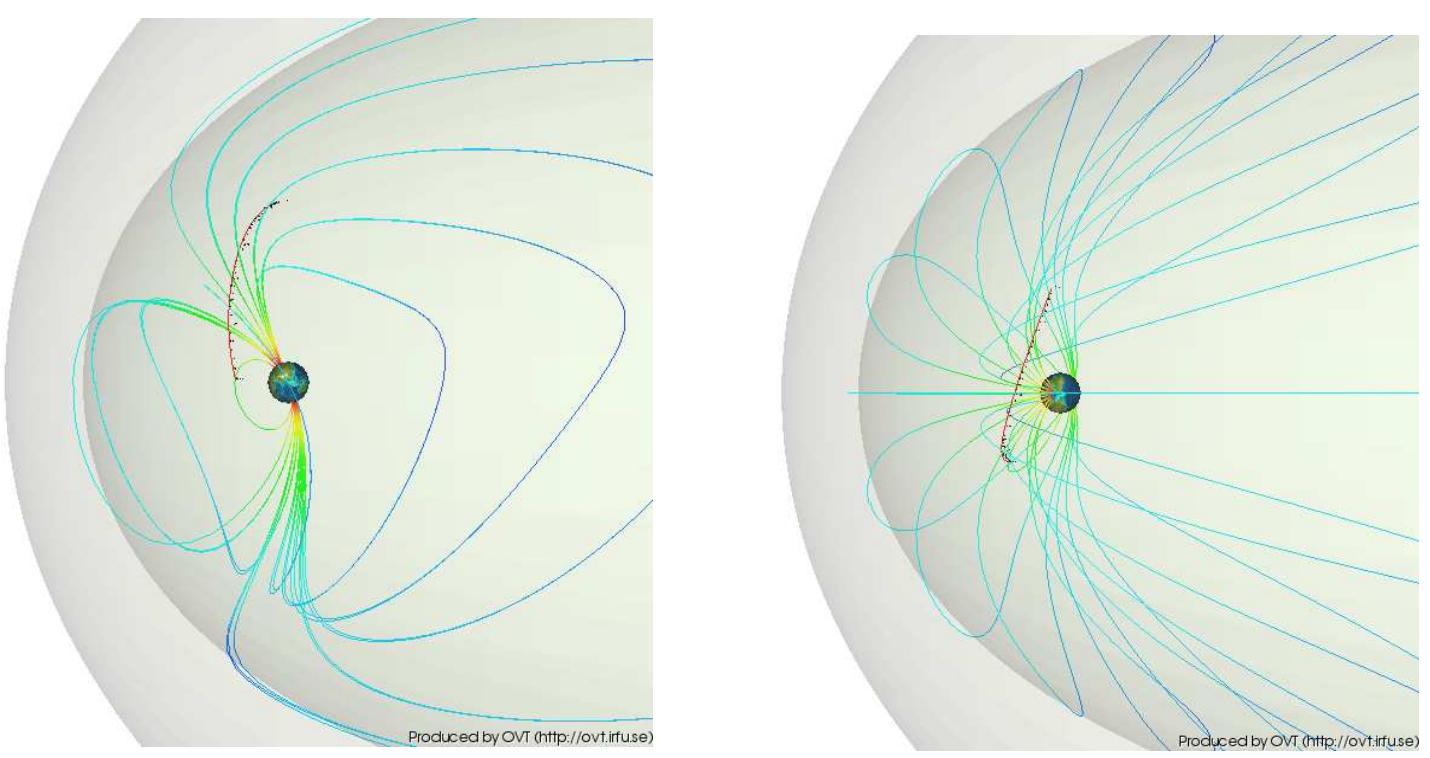

Fig. 5. Cluster orbit (red) for 4 July 2001, corresponding to the data in Fig. 6, viewed from GSE Y (left) and Z (right) directions. Model magnetosheath (light shading) and magnetosphere (dark shading) are shown, as are some magnetic field lines colour coded for magnetic field intensity. The Cluster motion is upward in both projections. Plot prepared using the Orbit Visualization Tool, http://ovt.irfu.se.

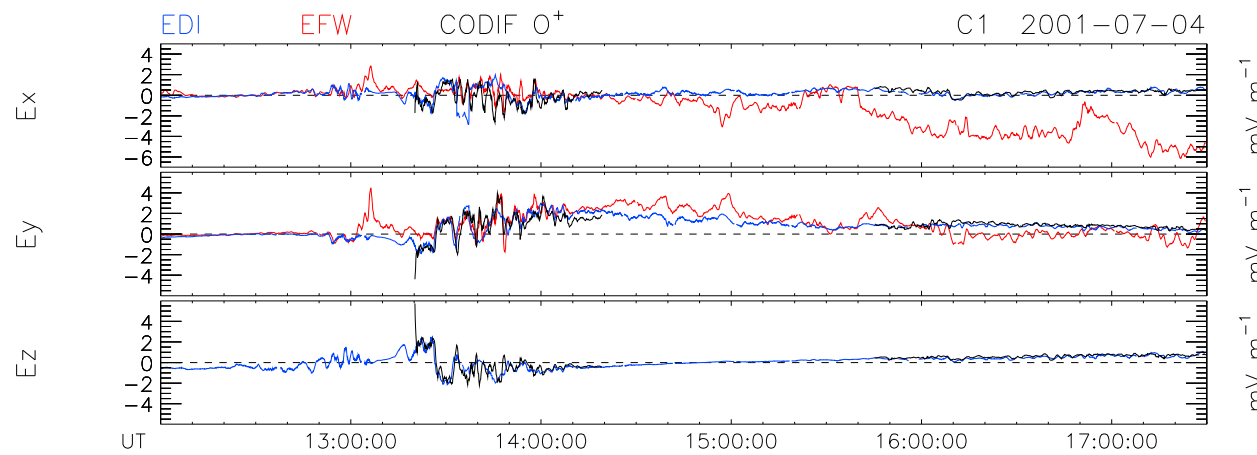

(a)

(b)

(c)

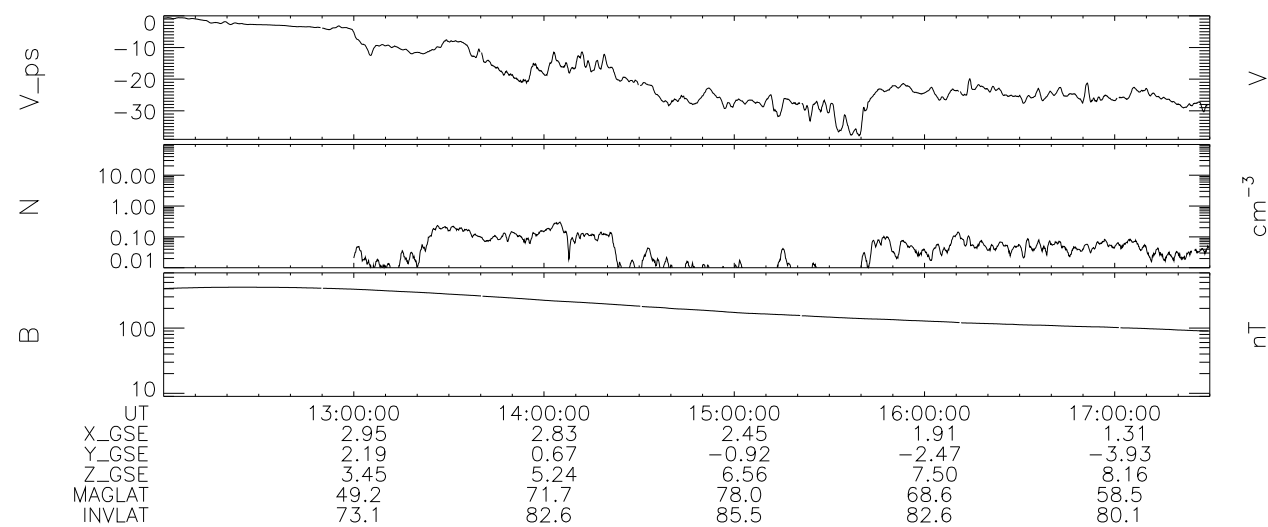

(d)

(e)

(f)

Fig. 6. Comparison of EFW and EDI data from the plasmasphere through the cusp/cleft into the polar cap. Format as in Fig. 4. 


\subsection{Example 2: Plasmasphere-boundary layer-polar cap}

\subsubsection{Geophysical setting}

Our second example is from 4 July 2001, between 12:00 and 17:30 UT. From the orbit plots in Fig. 5, we may expect Cluster to pass from the plasmasphere across boundary layer field lines into the polar cap. Data from Cluster SC1 are presented in Fig. 6, in a format similar to Fig. 4. Panel (d) at first shows $V_{\mathrm{ps}}$ values close to zero, corresponding to a plasmaspheric density above $100 \mathrm{~cm}^{-3}$. The plasmapause is crossed in a few minutes just before 12:15 UT, when the density as inferred from $V_{\mathrm{ps}}$ drops to $\sim 30 \mathrm{~cm}^{-3}$ in a region we may identify as the trough. The density decreases continuously to around $15 \mathrm{~cm}^{-3}$ at 13:00 UT, where another density drop signals a brief encounter with a part of the plasma sheet extending into the afternoon sector. The increased variations in the electric field, starting around 13:20 UT and continuing until after 14:00 UT, are consistent with the expectation that Cluster here should encounter boundary layer plasmas. Finally, the drop in hot plasma density, as seen by the CIS ion detectors (panel e) around 14:20 UT, signals the start of the open field line region of the polar cap, where the spacecraft remains for the rest of the time interval plotted.

\subsubsection{Inner magnetosphere}

In the plasmasphere and trough regions, i.e. 12:00-13:00 UT in Fig. 6, EFW and EDI are seen in panels (a) and (b) to agree to better than a $\mathrm{mV} / \mathrm{m}$ in this example, with the largest deviations seen in the plasmasphere (before 12:15 UT). A blowup of part of the trough region is seen in Fig. 7, showing detailed agreement to within $0.1 \mathrm{mV} / \mathrm{m}$ in the observation of pulsations, with periods around a minute. The EDI data have been filtered by a boxcar averager, but otherwise no corrections or filtering of any kind have been applied to the data.

Such good agreement is commonly found in the trough and subauroral regions, which generally are favourable to EDI and EFW alike. In the plasmasphere, there can sometimes be discrepancies due to the formation of plasma wakes (Bauer et al., 1983). However, we find a region of significant difference between EDI and EFW electric field measurements between 13:00 and 13:20 UT in Fig. 6. This will be discussed further in Sect. 3.

\subsubsection{Plasma sheet and boundary layer}

Let us first look at the time interval when Cluster encountered boundary layer field lines, i.e. around 13:20-14:00 UT in Fig. 6, as indicated by the higher level of electric field fluctuations. Here, the agreement between EDI and EFW as seen on this time scale again is very good. However, the boundary layer is a very dynamic region, and all dynamics certainly do not show up in this spin-resolution plot. Figure 8 again shows a blowup, with EFW data at full time resolution, which for this case was 25 samples/s. As is to be expected, EDI cannot adequately cover this dynamical situtation, though the data points actually acquired agrees well with EFW. As for

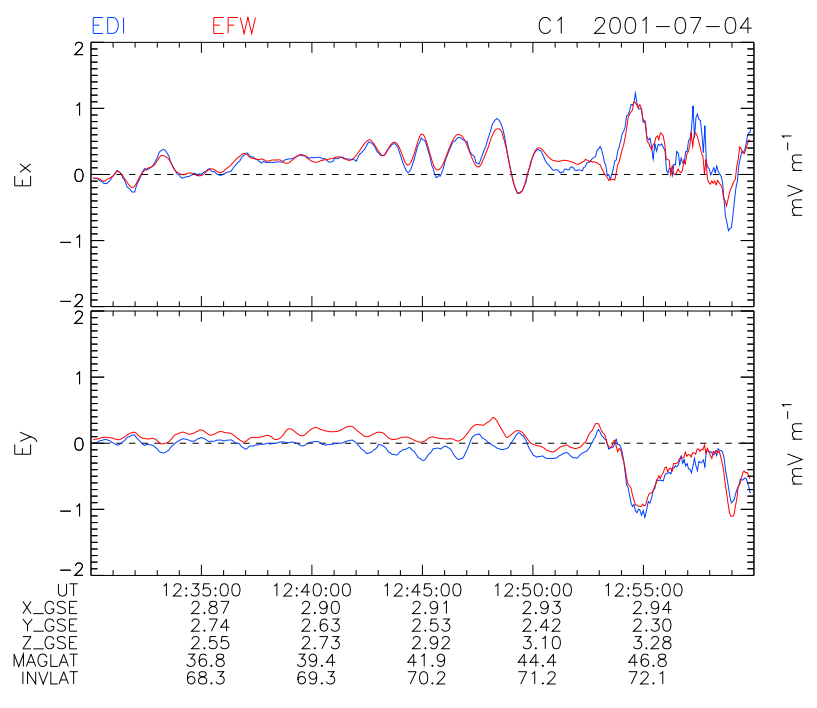

Fig. 7. Detail of part of the data in Fig. 6, showing agreement between EFW (red) and EDI (blue) to the level of a fraction of a $\mathrm{mV} / \mathrm{m}$ in the inner magnetosphere.

EFW, the good quality of the data shown here is common not only for boundary layer plasmas but is also dominating in the plasma sheet and auroral zone, and essentially always in the central plasma sheet (not shown). However, spurious fields can sometimes show up in double-probe data, as is illustrated by the large EDI-EFW discrepancy seen in the plasma sheet (Fig. 6, 13:00-13:20 UT). As was the case in the regions with some EDI-EFW discrepancy in Example 1 (Fig. 4), the plasma density indicated by the CIS instrument in this region (below $0.1 \mathrm{~cm}^{-3}$ ) is much lower than what is expected from the EFW $V_{\mathrm{ps}}$ value (around $1 \mathrm{~cm}^{-3}$ ), hinting that cold plasma may be the source of the problem. For the moment, we only note the existence of this kind of problem, which we will discuss in more detail in the following sections.

\subsubsection{Polar cap}

After leaving the boundary layer field lines around 14:20 UT (Fig. 6), the satellite enters the polar cap. The probe-tospacecraft potential $V_{\mathrm{ps}}$ of panel (d) stays between $-20 \mathrm{~V}$ and $-30 \mathrm{~V}$ for the remainder of the interval, indicating densities between $1 \mathrm{~cm}^{-3}$ and $0.3 \mathrm{~cm}^{-3}$, except for a brief excursion to $-40 \mathrm{~V}$ (around $0.1 \mathrm{~cm}^{-3}$ ) around 15:35 UT. Comparing to the CIS CODIF density moment in panel (e), it is clear that the density seen by the ion detector is only a small fraction of the total density, except possibly at the density minimum indicated by the EFW $V_{\mathrm{ps}}$ at 15:35 UT. In the polar cap region, the plasma component from the CIS data is readily identified as the polar wind, a cold plasma flow known to fill these regions in number densities comparable to those indicated by $V_{\mathrm{ps}}$. By using artificial potential control, the Polar spacecraft could be brought down to close to zero values of $V_{\mathrm{ps}}$, enabling the ions to reach the spacecraft and consequently allowing Moore et al. (1997) and Su et al. (1998) to determine 


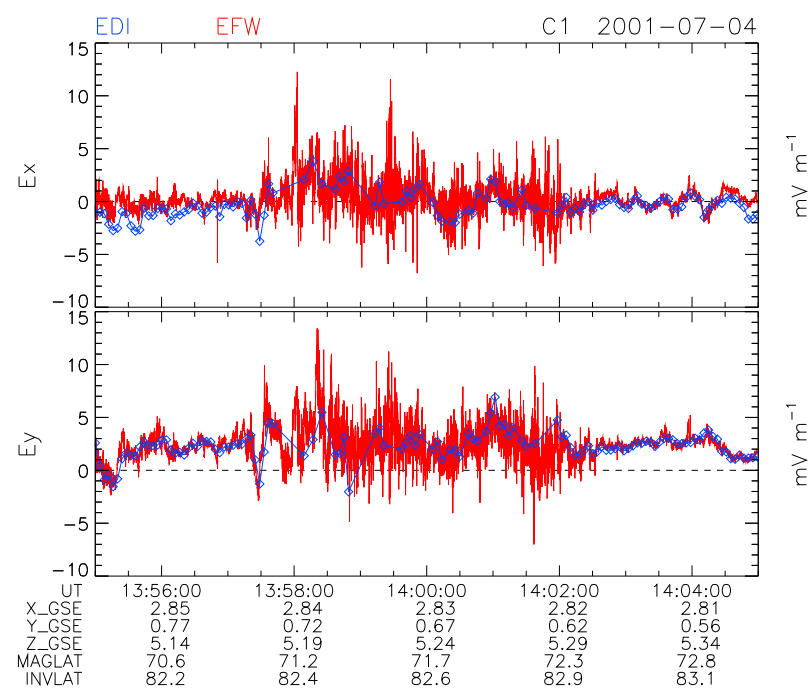

Fig. 8. Detail of part of the data in Fig. 6, with EFW (red) and EDI (blue) data at full time resolution in the plasma sheet boundary layer.

that the cold polar wind flow can be seen all the way out to the Polar apogee at $9 R_{E}$. Since the thermal energy, as well as the bulk flow energy of the ions in the polar wind, are below the typically observed spacecraft potential $-V_{\mathrm{ps}}$, we see why this plasma cannot reach the CIS detectors and hence escapes detection.

Differences between the electric field signals from EFW and EDI can be seen in panels (a) and (b) of Fig. 6. Although seen in the $Y$ component, the $X$ component is most significantly affected. EDI works well in this region, as can be seen by comparing to the E-field estimated from the CIS oxygen velocity moment when this is computable. At times, particularly around 15:30 UT, the data suggests some covariance between the EFW-EDI discrepancy and $V_{\mathrm{ps}}$. EDI, using $\mathrm{keV}$ electrons, should be insensitive to potential variations of the $10 \mathrm{~V}$ order, but this is certainly not the case for EFW. The indications thus point to the dominant measurement error originating from EFW rather than from EDI. The event presented here is not an isolated artifact: examples like this are commonly found in EFW-EDI comparisons in the polar cap region, and in preceeding sections in this paper we have seen similar discrepancies for briefer intervals in other regions, as well. It is obviously important to understand why: this will be the topic of Sect. 3 .

\subsection{Summary of events}

Summarizing what can be learned from the discussion around Figs. 4 and 6, we conclude that EFW produces good quality electric field data in the solar wind and the magnetosheath, with some spurious components on the order of a $\mathrm{mV} / \mathrm{m}$ often appearing in regions with a tenuous cold plasma component in the mantle. EDI produces no data at all in the solar wind and only intermittently in the magnetosheath, though the electric field estimates are usually good when present, particularly inside the magnetopause. In the plasmasphere and trough, the two instruments generally agree well, though EFW may sometimes pick up spurious signals, the nature of which we will return to in Sect. 3. This also happens at times in the auroral zone, though this region is usually more problematic for EDI than for EFW, as the strong and rapid electric field variations and the presence of intense auroral electrons may result in an EDI data loss. On the other hand, EDI provides very good data in the polar caps, at least sufficiently close to the Earth to keep the magnetic field above the EDI threshold of about $30 \mathrm{nT}$, where the EFW data often are severly contaminated or even dominated by spurious electric fields.

\section{Polar cap discrepancies}

\subsection{Spurious field and spacecraft potential}

Figure 9 shows a detailed view of $1 \mathrm{~h}$ of data from Fig. 6. Panel (a) shows $V_{\mathrm{ps}}$, approximately equal to the negative of the spacecraft potential $V_{\mathrm{sc}}$. During this hour, this quantity stays between $-20 \mathrm{~V}$ and $-30 \mathrm{~V}$, corresponding to plasma densities between about $1 \mathrm{~cm}^{-3}$ and $0.3 \mathrm{~cm}^{-3}$ (Pedersen et al., 2001), except for an excursion to $-15 \mathrm{~V}$, or $1.5 \mathrm{~cm}^{-3}$, at around $800 \mathrm{~s}$. Panels (b) and (c) show the $X$ and $Y$ components of the electric field from EDI (magenta) and EFW (red/black). The EFW data plotted are spin fits from probe pair 12 (red) and 34 (black). The data from the two probe pairs coincide nearly exactly, so that the black trace is hard to discern.

It can be seen in these panels (b) and (c) of Fig. 9, that EFW and EDI electric field measurements differ by several $\mathrm{mV} / \mathrm{m}$ during most of this interval. It is interesting to note that this discrepancy is the same regardless of the EFW probe pair used, as the red (P12) and black (P34) curves coincide. If it is the EDI field which is the more accurate representation of the unperturbed electric field in the plasma, the source of the perturbed field seen by EFW must be quite stable. That the field seen by EFW cannot only be the unperturbed electric field in the plasma should be clear from the EDI-CIS agreement shown previously in Fig. 6. A further indication is that the EFW E-field varies with the probe-to-spacecraft potential, $V_{\mathrm{ps}}$. This can be seen more clearly in panel (d), displaying the DSI $X$ (solid) and $Y$ (dashed) components of the difference between the instruments,

$\boldsymbol{E}_{\mathrm{spur}}=\boldsymbol{E}_{\mathrm{EFW}}-\boldsymbol{E}_{\mathrm{EDI}}$.

Comparing panel (d) to panel (a), we can immediately see that the difference between the instruments almost disappears at the temporary increases in $V_{\mathrm{ps}}$ at 800 and $3100 \mathrm{~s}$, and hints of a partial, albeit imperfect, covariation which can be seen during a large part of the plotted interval. As it is hard to conceive of a mechanism by which the EDI instrument, using electrons of keV energy, should be sensitive to potential variations of a few volts, this dependence on $V_{\mathrm{ps}}$ is independent 


\section{Cluster SC1 2001-07-04}

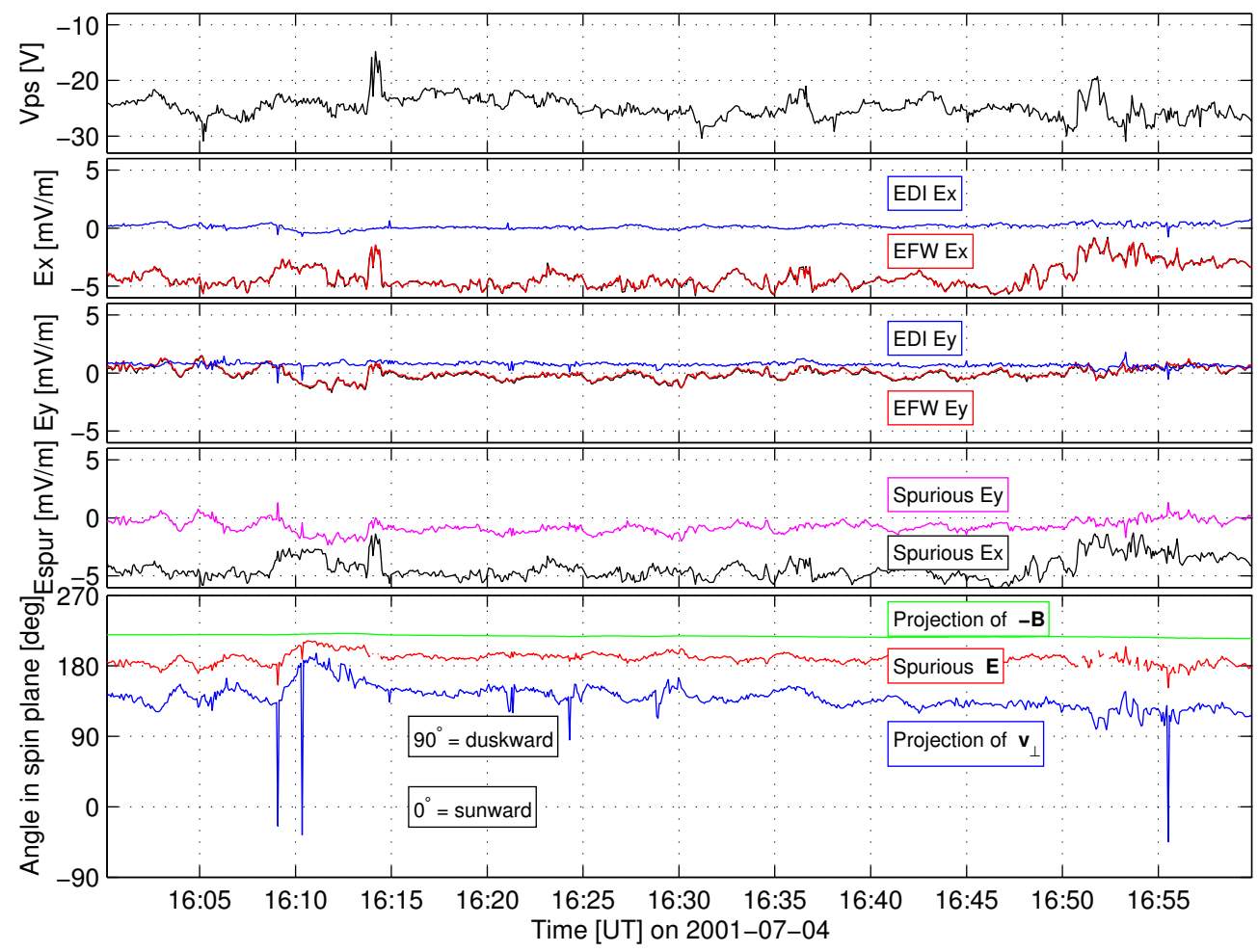

(a)

(b)

(c)

(d)

(e)

Fig. 9. Comparison of EFW and EDI data for an event with a spurious electric field in the EFW data. Panels from top to bottom: (a) EFW probe-to-spacecraft potential $V_{\mathrm{ps}} \approx-V_{\mathrm{sc}}$. (b) $E_{\mathrm{X}}$ (DSI coordinates, see text) in the spacecraft reference frame. Red and black represent EFW data based on spin fits from probe pairs 12 and 34, respectively, blue is EDI. (c) $E_{Y}$ in same format. (d) Spurious field in EFW data, assuming EDI is correct. Black is $E_{\mathrm{X}}$, magenta is $E_{\mathrm{Y}}$. (e) Angles of fields projected onto the spin plane, counted from the Sun direction, as shown in Fig. 10. Red is $E_{\mathrm{spur}}=\boldsymbol{E}_{\mathrm{EFW}}-\boldsymbol{E}_{\mathrm{EDI}}$, blue is the projection of EDI perpendicular drift velocity, green is the projection of the negative of the geomagnetic field (i.e. the direction along $\boldsymbol{B}$ pointing away from the Earth). The spike-like excursions (e.g. around 16:10 UT) originate from glitches in EDI, which are of no interest here.

evidence that the problem indeed is with the double-probe method. We thus conclude that the type of EFW-EDI discrepancy encountered in the polar cap is due to a spurious

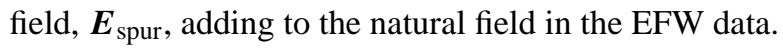

\subsection{Direction of spurious field}

To obtain further information on the spurious field, we will now study its direction by plotting a set of angles in the spin plane in panel (e) of Fig. 9. The directions are explained in Fig. 10: note that all angles are referring to projections in the spin plane, counted from the solar direction $\left(X_{\mathrm{DSI}} \approx X_{\mathrm{GSE}}\right)$ positive towards dusk $\left(Y_{\mathrm{DSI}} \approx Y_{\mathrm{GSE}}\right)$. The green line shows the angle of the projection of $-\boldsymbol{B}$ onto the spin plane: in the Northern Hemisphere, $-\boldsymbol{B}$ is the direction away from the Earth along the field lines. The angle of the projection of the EDI flow velocity, $\boldsymbol{v}_{\mathrm{EDI}}$, onto the spin plane is shown in blue. Note that while the full EDI flow velocity vector is necessarily perpendicular to $\boldsymbol{B}$ because of the EDI operational principle (Sect. 1), the projections of $\boldsymbol{v}_{\mathrm{EDI}}$ and $\boldsymbol{B}$ onto the spin plane do not need to be perpendicular.
The spin plane direction of the spurious E-field seen by EFW is shown in red. We can see that throughout the interval, this angle stays at around $180^{\circ}$, superficially suggesting that the spurious field may be antisunward. However, we may note that the direction of $\boldsymbol{E}_{\text {spur }}$ depends on the direction of the perpendicular part of the drift velocity $\boldsymbol{v}_{\perp}$ determined by EDI, shown in blue. In fact, the spurious field (red) always stays between $\boldsymbol{v}_{\perp}$ (blue) and $-\boldsymbol{B}$ (green). To determine which direction is more important, we show data from a Northern Hemisphere dawn-dusk orbit in Fig. 11, in the same format as in Fig. 9. Jumping directly to panel (e), we see that the spurious field stays between the $-\boldsymbol{B}$ and $\boldsymbol{v}_{\perp}$ also on this orbit, while it does not at all align with the solar direction. This is exactly the direction we expect for the polar wind plasma flow that is typical in this region of space: EDI should correctly pick up its perpendicular component $\boldsymbol{v}_{\perp}$ but cannot observe the parallel velocity component $\boldsymbol{v}_{\|}$. As the polar wind is an outflow along the geomagnetic field lines, the unobservable $\boldsymbol{v}_{\|}$should be antiparallel to the geomagnetic field, which here points toward the Earth. The polar wind velocity vector $\boldsymbol{v}_{\perp}+\boldsymbol{v}_{\|}$should thus lie between 


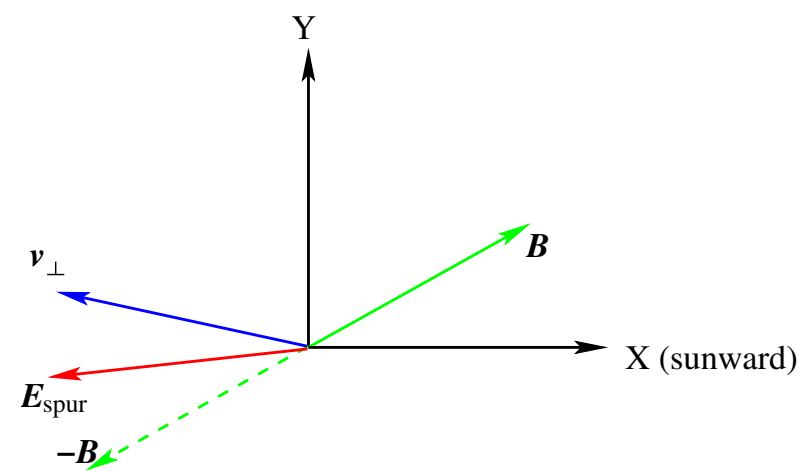

Fig. 10. Directions of various quantities projected onto the spin plane. $\mathrm{X}$ and $\mathrm{Y}$ are DSI coordinate axes, very close to the GSE axes, so that $\mathrm{X}$ is the solar direction which is a reference for the angles in Fig. 9. $\boldsymbol{B}$ is the ambient magnetospheric magnetic field, and $\boldsymbol{E}_{\text {spur }}$ is the spurious electric field. The projection of the perpendicular component of the plasma flow is denoted by $\boldsymbol{v}_{\perp}$. The projection in this plane of a 3-D flow velocity $\boldsymbol{v}$ with components perpendicular, as well as antiparallel to the magnetic field, would thus be directed between the $\boldsymbol{v}_{\text {perp }}$ and $-\boldsymbol{B}$ vectors, i.e. where we find $\boldsymbol{E}_{\text {spur }}$.

$-\boldsymbol{B}$ and $\boldsymbol{v}_{\perp}$, precisely as is the case of the observed spurious electric field. This strongly suggests that $\boldsymbol{E}_{\text {spur }}$ is related to the plasma flow. In the following section, we will discuss how such a spurious field may arise.

\subsection{Electrostatic wake model}

To understand the double-probe measurements, it is necessary to consider the potential in space around the spacecraft. Initially neglecting any background electric field, i.e. the field that we would like to measure, the electrostatic potential field $\Phi$ in the vicinity of the spacecraft will be determined by the spacecraft potential, $V_{\mathrm{sc}}$, and by any potentials induced in the plasma because of the presence of the satellite. In the following we will consider the possible contribution $\Phi_{\text {wake }}$ arising from a wake behind the spacecraft in a flowing plasma.

A wake is expected to form behind any object in a supersonic flow. In a plasma, where the thermal speed is usually much higher for electrons than for ions, wakes are usually negatively charged, as thermal motion will carry more electrons than ions into the wake. If the characteristic wake size $L$, which should be chosen to be in the direction where the wake is the thinnest, is around or exceeding the Debye length $\lambda_{D}$ in the surrounding plasma, negative potentials on the order of the thermal potential equivalent $K T_{\mathrm{e}} / e$ may appear,

$\Phi_{\text {wake }} \sim-\frac{K T_{\mathrm{e}}}{e}, \quad L \gtrsim \lambda_{\mathrm{D}}$

Values much above this cannot be reached, as electrons then cannot enter the wake, and consequently, charge accumulation stops. For $L \ll \lambda_{\mathrm{D}}$, a simple solution of Poisson's equa-

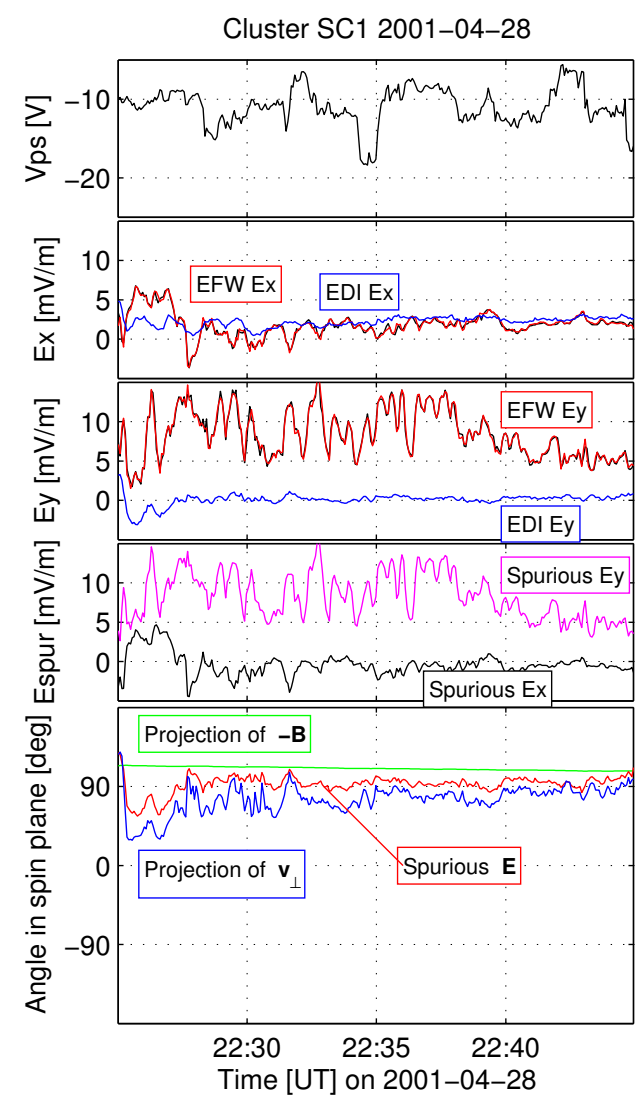

Fig. 11. Comparison of EFW and EDI data for an event with a spurious electric field in the EFW data, this time from a dawn-dusk orbit, in the same format as in Fig. 9.

tion for a planar slab structure, void of ions but with unperturbed electron density, suggests a scaling

$$
\Phi_{\text {wake }} \sim-\frac{K T_{\mathrm{e}}}{e}\left(\frac{L}{\lambda_{\mathrm{D}}}\right)^{2}, \quad L \ll \lambda_{\mathrm{D}}
$$

A slab geometry is more appropriate for an ion wake caused by an elongated absorbing physical target than for the repelling potential around a positively charged structure, for which we would rather expect ion deflection in a classical Rutherford scattering process. Nevertheless, an analogous scaling law, providing a rapid increase with size, will also apply in the deflection case. For Cluster, only in the cold and dense plasmasphere can the Debye length reach down to typical spacecraft dimensions, which can be taken to be the height or radius of the cylindrical spacecraft, i.e. $1-1.5 \mathrm{~m}$, and usually stays well above. The wakes forming behind a Cluster spacecraft, for example in the solar wind, could possibly be charged to a level of a volt or so, corresponding to some fraction of the solar wind electron temperature according to Eq. (3), but the influence that this wake, with a width of a meter or so, can have on the probes, $44 \mathrm{~m}$ away at the end of wire booms, must be small. Indeed, one can sometimes see a clear wake in EFW data from the solar wind, appearing as a brief spike in the data from each probe, once per spin, 
when the probe crosses the narrow wake (not shown). Such wake signatures are easily identifiable and cause few problems. The wire booms carrying the probes are only a few millimeters in diameter, so no significant potentials can build up in a wake caused by them. One may thus be tempted to conclude that wakes should not be much of a problem.

However, if the plasma is very tenuous, the spacecraft potential can be so high that the true obstacle to the ion flow is not the physical structure of the spacecraft, but the potential pattern surrounding it, which, to a first approximation, can be taken to be the vacuum potential arising from a satellite at potential $V_{\text {sc }}$. Thus, in the case

$K T_{\mathrm{i}}<\frac{1}{2} m_{\mathrm{i}} v_{\text {flow }}^{2}<e V_{\mathrm{sc}}$,

where $T_{\mathrm{i}}, m_{\mathrm{i}}$ and $v_{\text {flow }}$ are the ion temperature, mass and flow speed, $e$ is the elementary charge and $V_{\mathrm{sc}} \approx-V_{\mathrm{ps}}$ is the spacecraft potential, a wake will form whose characteristic size is determined not by the spacecraft or booms, but by the equipotential surface $\Phi=\frac{1}{2} m_{\mathrm{i}} v_{\text {flow }}^{2} / e$, as the ions will deflect before reaching this equipotential. In the cases of interest, the Debye length is much above the typical spacecraft scale size (a few meters), so the spacecraft potential is essentially a Coulomb field. For a spacecraft potential twice the ion flow energy, the $\Phi=\frac{1}{2} m_{\mathrm{i}} v_{\text {flow }}^{2} / e$ equipotential will thus be roughly one spacecraft radius away, increasing the effective cross section of the obstacle, as seen by the ion flow, by a factor of around $2^{2}=4$.

The formation of this kind of enhanced electrostatic wake around a spacecraft and its influence on the double-probe measurements on the GEOS and ISEE spacecraft was discussed by Bauer et al. (1983) and Pedersen et al. (1984). In comparisons to electron drift measurements and ion drift motion, they found that the wake formed by the $\boldsymbol{E} \times \boldsymbol{B}$ drift in a plasma caused perturbation of the measurement of $\boldsymbol{E}$. While they considered the increase in the effective size of the spacecraft, we should note that the effect may be even more dramatic around the wire booms, where the logarithmic potential decay applicable close to long booms can increase the effective obstacle cross section from millimeters to meters. In the case of very tenuous plasmas, $e V_{\mathrm{sc}} \gg \frac{1}{2} m_{\mathrm{i}} v_{\text {flow }}^{2} / e$, this wire-boom induced wake could be expected to be the more important contribution, while the spacecraft-induced wake should still dominate for $e V_{\mathrm{sc}} \gtrsim \frac{1}{2} m_{\mathrm{i}} v_{\text {flow }}^{2} / e$. The situation is illustrated qualitatively in Fig. 12b, where the shaded region indicates the negatively charged wake region, and also in Fig. 8 of Pedersen et al. (1984).

To further quantify these qualitative arguments for the formation of enhanced electrostatic wakes and their effects on double-probe measurements, we need numerical simulations of Cluster in a flowing plasma. Such particle-in-cell simulations (Engwall et al., 2004; Engwall, 2004) are indeed consistent with this hypothesis, showing a magnitude and angular dependence of the wake-induced electric field seen by EFW, agreeing well with our observations. A similar wake can also be seen in the simulations by Zinin et al. (2004). (a) $m v_{\mathrm{i}}^{2} / 2>K T_{\mathrm{i}}, m v_{\mathrm{i}}^{2} / 2>e V_{\mathrm{sc}}$

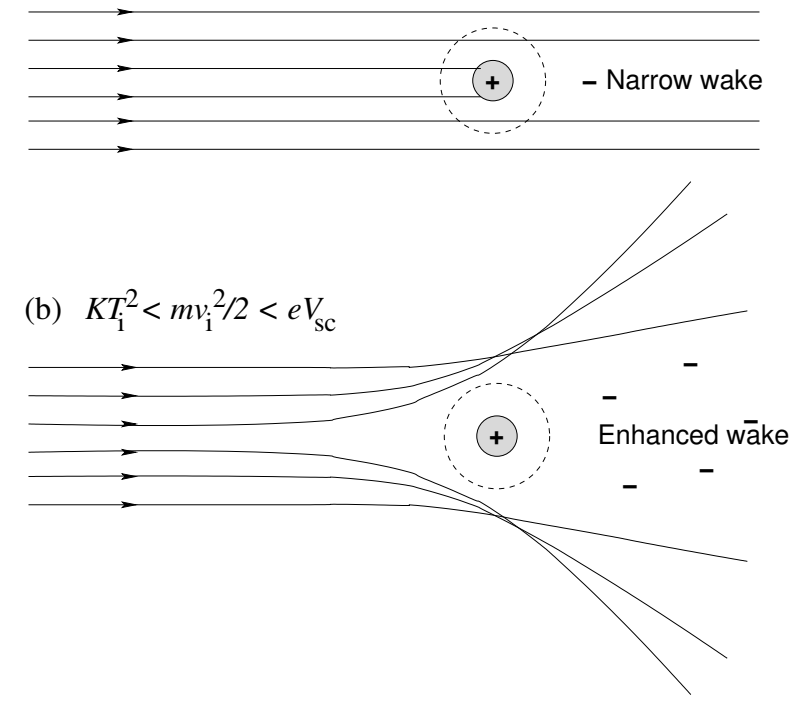

Fig. 12. Diagram illustrating ion wake formation behind a positive body subject to a supersonic ion flow from the left. The centre body could be the spacecraft itself, or a cut at right angles through a wire boom. (a) When the ion drift speed is higher than the spacecraft potential, the wake width is determined by the spacecraft geometric size. (b) When the potential is much higher than the ion flow energy, the ions deflect off the potential, so a measure of the effective obstacle size is set by the equipotential surface corresponding to the ion flow energy (dashed). The wake transverse size may thus be significantly larger than the geometrical dimensions of the body. In both cases, the wake is charged negatively by the random motion of the subsonic electrons.

\subsection{Effect of potential control}

Each Cluster satellite carries an instrument for artificial control of the spacecraft potential by the emission of ions, Active Spacecraft Potential Control (ASPOC) (Torkar et al., 2001). If the model presented above is correct, we expect the spurious electric field to depend on the spacecraft potential, and thus it should disappear, or at least decrease in magnitude, when ASPOC is used. Figure 13 shows that this is indeed the case. The figure shows data from two spacecraft, SC1 (upper three panels), on which ASPOC was not operational, and SC3 (lower three panels), with an operational ASPOC. In the SC1 data, we find that EFW (red) and EDI (blue) disagree strongly on the electric field after about 04:20 UT, indicating spurious electric fields sometimes exceeding $5 \mathrm{mV} / \mathrm{m}$. Between 04:20 and 04:24 UT, a similar spurious field can be seen to also emerge in the SC3 data. At 04:24 UT, ASPOC is turned on for $\mathrm{SC} 3$, which is immediately visible in the $V_{\mathrm{ps}}$ data shown in the bottom panel, as a sudden increase to a relatively steady value around $-7 \mathrm{~V}$. At the same time, the spurious electric field reduces drastically, particularly its $X$ component. Some difference between EDI and EFW remains even after 04:24 UT, but it is clear that the disagreement is less pronounced (around $1 \mathrm{mV} / \mathrm{m}$ ) than what we find on $\mathrm{SC} 1$ 

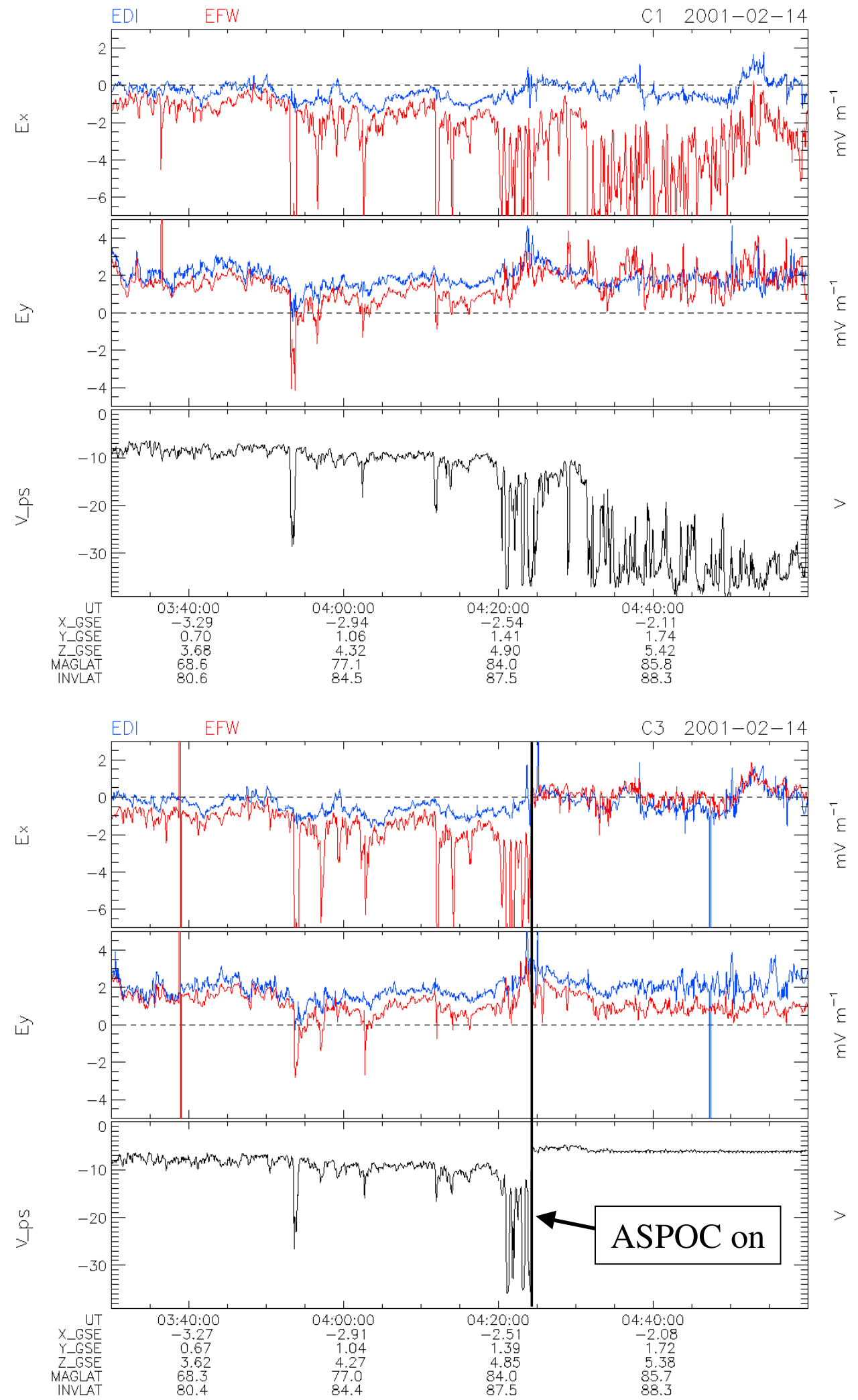

Fig. 13. Comparison of EFW (red) and EDI (blue) electric field spin plane components (DSI coordinates, close to GSE) for an event with a spurious electric field in the EFW data, for SC1 and SC3. On SC3, the artificial potential controller ASPOC is turned on at 04:24 UT, immediately alleviating the spurious $E_{\mathrm{X}}$ in the EFW data on this spacecraft. 
(often 3 to $5 \mathrm{mV} / \mathrm{m}$ ). This is clearly the behaviour we expect from the wake model: the wake electric field should decrease in amplitude when the spacecraft potential drops, but it does not have to disappear completely, since even when ASPOC is on, the spacecraft remains positive at more than $7 \mathrm{~V}$ (one should add the potential drop over the probe sheath of around $1 \mathrm{~V}$ to $-V_{\mathrm{ps}}$ ). This is not an isolated example: the behaviour is consistent in all cases examined. Together with the directional considerations in Sect. 3.2, we interpret this as evidence for the electrostatic wake model.

\subsection{Implications for other regions}

Up to now, all of Sect. 3 has considered the polar wind plasma. However, the enhanced electrostatic wake mechanism outlined in Sect. 3.3, of course, works in other regions with a cold plasma present. When considering the two example orbits in Sect. 2, we found several examples of EDI and EFW electric field estimates deviating from each other, and we also found that in these cases, the EFW $V_{\mathrm{ps}}$ measurment indicates the presence of a significant or even dominant component of cold plasma not seen by the ion instrument CIS, and hence with an energy below $e V_{\text {sc }}$. If this plasma is flowing sufficiently fast, we will obtain exactly the situation $K T_{\mathrm{i}}<\frac{1}{2} m_{\mathrm{i}} v_{\text {flow }}^{2}<e V_{\mathrm{sc}}$, where an enhanced wake is expected to develop, and significant spurious fields appear, explaining why EFW-EDI discrepancies tend to turn up in regions where there is a significant cold plasma population not seen by CIS.

The cold plasma of the polar wind does not stay close to the Earth. Detailed observations from the Polar satellite established its properties at $9 R_{E}$ (Moore et al., 1997; $\mathrm{Su}$ et al., 1998). Recently, Sauvaud et al. (2004) have shown several examples of cold plasma in the tail out to $18 R_{E}$. The polar wind is only one of the magnetospheric cold plasma populations. Our example orbits (Sect. 2) show that cold plasma can indeed turn up elsewhere, as has been also noted in other studies. By using the ISEE-1 relaxation sounder, Etcheto and Saint-Marc (1985) found a plasma component below $30 \mathrm{eV}$ sometimes dominating the plasma sheet boundary layer, reaching densities of $5 \mathrm{~cm}^{-3}$. More recently, Seki et al. (2003) found a similar plasma component in Geotail data from the plasma sheet itself, where we clearly had spurious fields in our example (Sect. 2.2.3). Cold plasma originating from plasmaspheric detachments have been reported in the magnetosphere in several studies (e.g. Chappell, 1974; Elphic et al., 1996; Matsui et al., 1999; Foster et al., 2004), mainly on the dayside, but it may also propagate to the tail (Elphic et al., 1997). Of particular interest are the reports from Cluster (Sauvaud et al., 2001) and Polar (Chen and Moore, 2004) on cold plasmas with density $\sim 1 \mathrm{~cm}^{-3}$ and temperature below $10 \mathrm{eV}$ just inside the magnetopause. In this region, densities are low and spacecraft potentials are correspondingly high, often much above $10 \mathrm{~V}$, but in the published cases, the ions nevertheless had sufficient energy to reach the ion detectors on the spacecraft because of their high flow speed, $\sim 150 \mathrm{~km} / \mathrm{s}$. In a situation with lower flow speed and/or higher spacecraft potential, the ions may go unnoticed and cause the type of wake effects we have discussed above for the polar wind.

\section{Conclusions}

In this paper, we have reported on comparisons of EDI and EFW instruments from different plasma regions, illustrating them with some example events. A summary is given in Sect. 2.3. Our main conclusions are as follows:

1. The general performance of both instruments is good, with particular merits and drawbacks, as illustrated in Table 1.

2. For Cluster, the limitations on EDI mainly show up as periods when no E-field can be derived. When EDI data are present, they are generally good, except in dynamic regions like the auroral zone, where random aliasinglike effects may occur.

3. EFW, on the other hand, provides data in all environments and to high frequencies, but the DC electric fields derived can, in some environments, be contaminated by nongeophysical signals.

4. Double-probe electric field data can be contaminated by local fields arising from enhanced electrostatic wakes in regions where $K T_{\mathrm{i}}<\frac{1}{2} m_{\mathrm{i}} v_{\text {flow }}^{2}<e V_{\text {sc }}$. On Cluster, this mainly happens in the polar wind but sometimes also in other regions.

5. If the plasma density estimate from ion spectrometer moments gives lower values than expected from spacecraft potential or plasma frequency measurements, this can be an indication that there may be nongeophysical electric fields in double-probe data caused by an enhanced cold plasma wake. A lower density in the ion moments indicates plasma with energy below the s/c potential, and hence a risk for wide wakes and wakeinduced electric fields.

6. On Cluster, the problem of enhanced electrostatic wakes in flowing cold plasma is alleviated (but not eliminated) by the use of artificial spacecraft potential control.

7. The double-probe and electron drift techniques for measuring the electric field are complementary to each other. The Cluster spacecraft, carrying both kinds of instruments, are well equipped to measure the electric field in all regions.

Can electric field data from double-probe instruments like EFW be cleaned from the effects of spacecraft wakes? Removing narrow wakes, such as those sometimes encountered in the solar wind (Fig. 12a), can be done on a routine basis, but the wide enhanced wakes we have discussed in Sect. 3.3 are more difficult to correct, since their signature is quite similar to that of a large-scale electric field and is often dominant 
in the data. A technique based on establishing relations between the spin harmonics in the high-resolution data is attempted by Engwall and Eriksson (2005), but it is not clear if this method can be made practical for routine analysis. However, by using the information from CIS and EDI for determining offsets and wake effects at low frequencies, EFW data can clearly achieve high accuracy over a wide frequency range, even in cases where wake effects would otherwise cause problems. Frequencies above the spin frequency are not greatly affected by wide wakes, so it is possible to combine spin-resolution data from EDI with higher frequency measurements, to obtain accurate high-resolution data, even if no other data are available for comparison. Comparisons to EDI and CIS/FGM data are thus included in the preparation of EFW data for the Cluster Active Archive (Lindqvist et al., 2005), which also includes a data product containing electric fields filtered above the first spin harmonics.

A well understood wake effect is not only a problem: it is also a means to measure properties of the plasma causing the wake. Recently, Engwall et al. $(2005)^{2}$ have demonstrated that it is indeed possible to derive polar wind flow speed from the wake information obtained by combining EFW and EDI data.

Acknowledgements. Many thanks to E. Engwall for very useful discussion and cooperation. Data from the Cluster FGM and CIS instruments have been used with kind permission from the respective principal investigators, A. Balogh (succeeded by E. Lucek) and H. Rème.

Topical Editor T. Pulkkinen thanks K. Tsuruda and N. Cornilleau-Wehrlin for their help in evaluating this paper.

\section{References}

Balogh, A., Carr, C. M., Acuña, M. H., Dunlop, M. W., Beek, T. J., Brown, P., Fornaçon, K.-H., Georgescu, E., Glassmeier, K.-H., Harris, J., Musmann, G., Oddy, T., and Schwingenschuh, K.: The Cluster magnetic field investigation: overview of in-flight performance and initial results, Ann. Geophys., 19, 1207-1217, 2001,

SRef-ID: 1432-0576/ag/2001-19-1207.

Bauer, O. H., Grard, R., Haerendel, G., and Pedersen, A.: Influence of wake and photoemission on electric field measurements with a double probe on GEOS-2, Spacecraft/Plasma Interactions and their Influence on Field and Particle Measurements, Proceedings of the 17th ESLAB Symposium, ESA-SP, 51-56, 1983.

Chappell, C. R.: Detached plasma regions in the magnetosphere, J. Geophys. Res., 79, 1861-1870, 1974.

Chen, S.-H. and Moore, T. E.: Dayside flow bursts in the Earth's outer magnetosphere, J. Geophys. Res., 109, A03 215, doi:10.1029/2003JA010007, 2004.

Daly, P. W.: Users guide to the Cluster science data system, Tech. Rep. DS-MPA-TN-0015 (http://sci2.estec.esa.nl/cluster/ csds/csds.html), Max-Planck-Institut für Aeronomie, Lindau, Germany, 2002.

\footnotetext{
${ }^{2}$ Engwall, E., Eriksson, A. I., André, M., Dandouras, I., Paschmann, G., Quinn, J., and Torkar, K.: Low-energy (order $10 \mathrm{eV}$ ) ion flow in the magnetotail lobes inferred from spacecraft wake observations, Geophys. Res. Lett., in review, 2005.
}

Elphic, R. C., Weiss, L. A., Thomsen, M. F., McComas, D. J., and Moldwin, M. B.: Evolution of plasmaspheric ions at geosynchronous orbit during times of high geomagnetic activity, Geophys. Res. Lett., 23, 2189-2192, 1996.

Elphic, R. C., Thomsen, M. F., and Borovsky, J. E.: The fate of the outer plasmasphere, Geophys. Res. Lett., 24, 365-368, 1997.

Engwall, E.: Numerical Studies of Spacecraft-Plasma Interaction: Simulations of Wake Effects on the Cluster Electric Field Instrument EFW, IRF Scientific Report 284, Swedish Institute of Space Physics, Uppsala (http://www.irf.se/Publications/ IRFreport284.pdf), 2004.

Engwall, E. and Eriksson, A. I.: Cold magnetospheric plasma flows and spacecraft wakes: PicUp3D simulations and Cluster data, in: Proceedings of the 9th International Spacecraft Science Technology Conference (SCTC-9), Japan Aerospace Exploration Agency, 2005.

Engwall, E., Eriksson, A. I., Pedersen, A., Forest, J., Paschmann, G., Quinn, J., Torbert, R., and Torkar, K.: Wake effects on positively charged spacecraft in flowing tenuous plasmas: Cluster observations and modelling, in Proceedings of the 8th Spacecraft Charging Technology Conference, Huntsville, October 2003, NASA, 2004.

Escoubet, C. P., Fehringer, M., and Goldstein, M.: The Cluster mission, Ann. Geophys., 19, 1197-1200, 2001,

SRef-ID: 1432-0576/ag/2001-19-1197.

Etcheto, J. and Saint-Marc, A.: Anomalously high plasma densities in the plasma sheet boundary layer, J. Geophys. Res., 90, 53385344, 1985.

Foster, J. C., Coster, A. J., Erickson, P. J., Rich, F. J., and Sandel, B. R.: Stormtime observations of the flux of plasmaspheric ions to the dayside cusp/magnetopause, Geophys. Res. Lett., 31, doi:10.1029/2004GL020082, 2004.

Gustafsson, G., Boström, R., Holback, B., Holmgren, G., Lundgren, A., Stasiewicz, K., Ahlén, L., Mozer, F. S., Pankow, D., Harvey, P., Berg, P., Ulrich, R., Pedersen, A., Schmidt, R., Butler, A., Fransen, A. W. C., Klinge, D., Thomsen, M., Fälthammar, C.G., Lindqvist, P.-A., Christenson, S., Holtet, J., Lybekk, B., Sten, T. A., Tanskanen, P., Lappalainen, K., and Wygant, J.: The electric field and wave experiment for the Cluster mission, Space. Sci. Rev., 79, 137-156, 1997.

Gustafsson, G., André, M., Carozzi, T., Eriksson, A. I., Fälthammar, C.-G., Grard, R., Holmgren, G., Holtet, J. A., Ivchenko, N., Karlsson, T., Khotyaintsev, Y., Klimov, S., Laakso, H., Lindqvist, P.-A., Lybekk, B., Marklund, G., Mozer, F., Mursula, K., Pedersen, A., Popielawska, B., Savin, S., Stasiewicz, K., Tanskanen, P., Vaivads, A., and Wahlund, J.-E.: First results of electric field and density observations by Cluster EFW based on initial months of operation, Ann. Geophys., 19, 1219-1240, 2001,

SRef-ID: 1432-0576/ag/2001-19-1219.

Harvey, P., Mozer, F. S., Pankow, D., Wygant, J., Maynard, N. C., Singer, H., Sullivan, W., Anderson, P. B., Pfaff, R., Aggson, T., Pedersen, A., Fälthammar, C.-G., and Tanskanen, P.: The electric field instrument on the Polar satellite, Space Sci. Rev., 71, 583596, 1995.

Kletzing, C. A., Paschmann, G., Boehm, M. H., Haerendel, G., Sckopke, N., Baumjohann, W., Torbert, R. B., Marklund, G., and Lindqvist, P.-A.: Electric fields derived from electron drift measurements, Geophys. Res. Lett., 21, 1863-1966, 1994.

Lindqvist, P.-A., Khotyaintsev, Y., André, M., and Eriksson, A. I.: EFW data in the Cluster active archive, in: Proceedings of the Cluster and Double Star symposium: 5th anniversary of Cluster 
in space, ESA, in press, 2005.

Matsui, H., Mukai, T., Ohtani, S., Hayashi, K., Elphic, R. C., Thomsen, M. F., and Matsumoto, H.: Cold dense plasma in the outer magnetosphere, J. Geophys. Res., 104, 25 077-25 096, 1999.

Maynard, N. C.: Electric field measurements in moderate to high density space plasmas with passive double probes, in: Measurement Techniques in Space Plasmas: Fields (AGU Geophysical Monograph, 103), edited by: Borovsky, J., Pfaff, R., and Young, D., American Geophysical Union, 13-27, 1998.

Moore, T. E., Chappell, C. R., Chandler, M. O., Craven, P. D., Giles, B. L., Pollock, C. J., Burch, J. L., Young Jr., D. T., Hunter Waite, J., Nordholt, J. E., Thomsen, M. F., McComas, D. J., Berthelier, J. J., Williamson, W. S., Robson, R., and Mozer, F. S.: Highaltitude observations of the polar wind, Science, 277, 349-351, 1997.

Mozer, F. S.: Analyses of techniques for measuring DC and AC electric fields in the magnetosphere, Space Sci. Rev., 14, 272 313, 1973.

Paschmann, G., Melzner, F., Frenzel, R., Vaith, H., Parigger, P., Pagel, U., Bauer, O., Haerendel, G., Baumjohann, W., Sckopke, N., Torbert, R., Briggs, B., Chan, J., Lynch, K., Morey, K., Quinn, J., Simpson, D., Young, C., McIlwain, C., Fillius, W., Kerr, S., Mahieu, R., and Whipple, E.: The Electron Drift Instrument for Cluster, Space Sci. Rev. , 79, 233-269, 1997.

Paschmann, G., McIlwain, C. E., Quinn, J. M., Torbert, R. B., and Whipple, E. C.: The electron drift technique for measuring electric and magnetic fields, in: Measurement Techniques in Space Plasmas: Fields (AGU Geophysical Monograph, 103), edited by: Borovsky, J., Pfaff, R., and Young, D., American Geophysical Union, 29-38, 1998.

Paschmann, G., Quinn, J. M., Torbert, R. B., Vaith, H., McIlwain, C. E., Haerendel, G., Bauer, O. H., Bauer, T., Baumjohann, W., Fillius, W., Förster, M., Frey, S., Georgescu, E., Kerr, S. S., Kletzing, C. A., Matsui, H., Puhl-Quinn, P., and Whipple, E. C.: The Electron Drift Instrument on Cluster: overview of first results, Ann. Geophys., 19, 1273-1288, 2001,

SRef-ID: 1432-0576/ag/2001-19-1273

Pedersen, A., Cattell, C. A., Fälthammar, C.-G., Formisano, V., Lindqvist, P.-A., Mozer, F., and Torbert, R.: Quasistatic electric field measurements with spherical double probes on the GEOS and ISEE satellites, Space Sci. Rev., 37, 269-312, 1984.

Pedersen, A., Mozer, F., and Gustafsson, G.: Electric field measurements in a tenuous plasma with spherical double probes, in: Measurement Techniques in Space Plasmas: Fields (AGU Geophysical Monograph, 103), edited by: Borovsky, J., Pfaff, R., and Young, D., American Geophysical Union, 1-12, 1998.

Pedersen, A., Decreau, P., Escoubet, P., Gustafsson, G., Laakso, H., Lindqvist, P.-A., Lybekk, B., Mozer, F., and Vaivads, A.: Fourpoint high time resolution information on electron densities on Cluster, Ann. Geophys., 19, 1471-1481, 2001,

SRef-ID: 1432-0576/ag/2001-19-1471.

Rème, H., Aoustin, C., Bosqued, J. M., Dandouras, I., Lavraud, B., Sauvaud, J. A., Barthe, A., Bouyssou, J., Camus, T., Coeur-Joly, O., Cros, A., Cuvilo, J., Ducay, F., Garbarowitz, Y., Medale, J. L., Penou, E., Perrier, H., Romefort, D., Rouzaud, J., Vallat, C., Alcaydé, D., Jacquey, C., Mazelle, C., d'Uston, C., Möbius, E., Kistler, L. M., Crocker, K., Granoff, M., Mouikis, C., Popecki, M., Vosbury, M., Klecker, B., Hovestadt, D., Kucharek, H., Kuenneth, E., Paschmann, G., Scholer, M., Sckopke, N., Seidenschwang, E., Carlson, C. W., Curtis, D. W., Ingraham, C., Lin, R. P., McFadden, J. P., Parks, G. K., Phan, T., Formisano, V., Amata, E., Bavassano-Cattaneo, M. B., Baldetti, P., Bruno,
R., Chionchio, G., Lellis, A. D., Marcucci, M. F., Pallocchia, G., Korth, A., Daly, P. W., Graeve, B., Rosenbauer, H., Vasyliunas, V., McCarthy, M., Wilber, M., Eliasson, L., Lundin, R., Olsen, S., Shelley, E. G., Fuselier, S., Ghielmetti, A. G., Lennartsson, W., Escoubet, C. P., Balsiger, H., Friedel, R., Cao, J.-B., Kovrazhkin, R. A., Papamastorakis, I., Pellat, R., Scudder, J., and Sonnerup, B.: First multispacecraft ion measurements in and near the Earth's magnetosphere with the identical Cluster ion spectrometry (CIS) experiment, Ann. Geophys., 19, 1303-1354, 2001,

SRef-ID: 1432-0576/ag/2001-19-1303.

Sauvaud, J.-A., Lundin, R., Rème, H., McFadden, J., Carlson, C., Parks, G., Möbius, E., Kistler, L. M., Klecker, B., Amata, E., DeLellis, A. M., Formisano, V., Bosqued, J. M., Dandouras, I., Décreau, P., Dunlop, M., Eliasson, L., Korth, A., Lavraud, B., and McCarthy, M.: Intermitent thermal plasma acceleration linked to sporadic motions of the magnetopause: first Cluster results, Ann. Geophys., 19, 1523-1532, 2001,

SRef-ID: 1432-0576/ag/2001-19-1523.

Sauvaud, J.-A., Louarn, P., Fruit, G., Stenuit, H., Vallat, C., Dandouras, J., Rème, H., André, M., Balogh, A., Dunlop, M., Kistler, L., Möbius, E., Mouikis, C., Klecker, B., Parks, G. K., McFadden, J., Carlson, C., Marcucci, F., Pallocchia, G., Lundin, R., Korth, A., and McCarthy, M.: Case studies of the dynamics of ionospheric ions in the Earth's magnetotail, J. Geophys. Res., 109, doi:10.1029/2003JA009996, 2004.

Seki, K., Hirahara, M., Hoshino, M., Terasawa, T., Elphic, R. C., Saito, Y., Mukai, T., Hayakawa, H., Kojima, H., and Matsumoto, H.: Cold ions in the hot plasma sheet of Earth's magnetotail, Nature, 422, 589-592, 2003.

Su, Y.-J., Horwitz, J. L., Moore, T. E., Giles, L., Chandler, M. O., Craven, P. D., Hirahara, M., and Pollock, C. J.: Polar wind survey with the thermal ion dynamics experiment/plasma source instrument suite aboard POLAR, J. Geophys. Res., 103, 29305 29337, 1998.

Torkar, K., Riedler, W., Escoubet, C. P., Fehringer, M., Schmidt, R., Grard, R. J. L., Arends, H., Rüdenauer, F., Steiger, W., Narheim, B. T., Svenes, K., Torbert, R., André, M., Fazakerley, A., Goldstein, R., Olsen, R. C., Pedersen, A., Whipple, E., and Zhao, H.: Active spacecraft potential control for Cluster - implementation and first results, Ann. Geophys., 19, 1289-1302, 2001,

SRef-ID: 1432-0576/ag/2001-19-1289.

Tsuruda, K., Hayakawa, H., Nakamura, M., Okada, T., Matsuoka, A., Mozer, F. S., and Schmidt, R.: Electric field measurments on the GEOTAIL satellite, J. Geomag. Geoelectr., 46, 693-711, 1994.

Zinin, L., Grigoriev, S., and Rylina, I.: The models of electric field distributions near satellite, in: Auroral phenomena and solarterrestrial relations: Proceedings of the conference in memory of Yuri Galperin, edited by: Zelenyi, M. L. M. and Allen, J. H., CAWSES Handbook-001, 76-83, 2004. 